\title{
Conversations au sein de l'Atlantique noir. Ou comment les diasporas créent leurs mères patries.
}

À propos de :

GILROY Paul, L'Atlantique noir. Modernité et double conscience. Paris, Kargo, 2003, 235 p. (trad. de The Black Atlantic. Modernity and double consciousness, 1993, Cambridge, Harvard University Press)

MATORY J. Lorand, Black Atlantic Religion : Tradition, Transnationalism, and Matriarchy in the Afro-Brazilian Candomblé. Princeton, Princeton University Press, 2005, VIII +383 p.

\section{Stefania Capone}

\section{(2) OpenEdition}

Journals

Édition électronique

URL : http://journals.openedition.org/assr/3825

DOI : $10.4000 /$ assr.3825

ISSN : $1777-5825$

Éditeur

Éditions de l'EHESS

Édition imprimée

Date de publication : 1 décembre 2006

Pagination : 93-102

ISBN : 2-7132-2124-2

ISSN : 0335-5985

Référence électronique

Stefania Capone, « Conversations au sein de l'Atlantique noir. Ou comment les diasporas créent leurs mères patries. ", Archives de sciences sociales des religions [En ligne], 136 | octobre - décembre 2006, mis en ligne le 07 février 2007, consulté le 03 mai 2019. URL : http://journals.openedition.org/ assr/3825; DOI : 10.4000/assr.3825 


\title{
Stefania Capone \\ Conversations au sein de l'Atlantique noir Ou comment les diasporas créent leurs mères patries
}

\author{
À propos de : \\ Gilroy Paul, L'Atlantique noir. Modernité et double conscience. \\ Paris, Kargo, 2003, 235 p. (trad. de The Black Atlantic. Modernity \\ and double consciousness, 1993, Cambridge, Harvard University \\ Press). \\ Matory J. Lorand, Black Atlantic Religion: Tradition, Transnationa- \\ lism, and Matriarchy in the Afro-Brazilian Candomblé. Princeton, \\ Princeton University Press, 2005, VIII +383 p.
}

En 1993, Paul Gilroy publiait un des ouvrages qui ont le plus influencé le domaine afroaméricaniste, au moins dans les pays anglo-saxons: The Black Atlantic. Modernity and double consciousness. Avec ce livre, il a réussi à imposer une notion, celle d' "Atlantique noir ", devenue de nos jours un outil formidable pour penser les rapports triangulaires entre les Amériques, l'Afrique et l'Europe. Cette idée d'un espace - géographique, culturel et commercial - dans lequel se sont formées de nouvelles cultures et qui constitue, pour reprendre l'expression d'Immanuel Wallerstein ${ }^{1}$, un véritable "système-monde », est aussi le produit d'un changement de perspective qui a bouleversé en profondeur la discipline anthropologique. Ainsi, depuis au moins les années soixante, une nouvelle approche a remis en question l'idée même d'isolat " primitif », des isolats qui devaient être étudiés avant leur irrémédiable disparition, entraînée par l'irruption de la "modernité ". Le modèle fondé sur l'idée de l'existence de "systèmes délimités » a ainsi été remplacé par une approche dynamique des sociétés étudiées, dans laquelle sont mis en avant les liens et les échanges entre différentes cultures et sociétés.

Certains anthropologues, inspirés par les recherches d'I. Wallerstein sur le "système-monde " et par celles de Fernand Braudel sur une histoire sociale des grands réseaux d'échanges économiques, notamment dans la Méditerranée, se

1. Immanuel Wallerstein, Capitalisme et économie-monde, 1450-1640, Paris, Flammarion, 1980 
sont donc progressivement intéressés à la migration des personnes et des choses dans une « économie globale ». Des historiens, comme Curtin ${ }^{2}$ et al. ou Thornton ${ }^{3}$, ont montré l'importance de ces champs translocaux qui ont rendu possible la rencontre entre différents peuples et cultures. Cette idée a été déclinée de différentes façons : Philip Curtin parle d'un "South Atlantic system ", tandis que John Thornton développe la notion d' «Atlantic world» comme son principal outil d'analyse. Ce n'est pas par hasard s'il s'agit là d'historiens de la traite négrière et des liens entre Afrique et Amériques. On retrouve, en fait, dans cette approche une nouvelle réactivation de ce « commerce triangulaire » à l'origine de la colonisation du continent américain et de la déportation de grands contingents d'Africains, réduits en esclavage. En 1983, l'historien de l'art Robert Farris Thompson ${ }^{4}$ a été le premier à employer l'expression "Black Atlantic» en référence à une supposée continuité entre les cultures africaines et afro-américaines. Bien d'autres auteurs le suivront sur ce chemin, essayant de démontrer l'existence d'une « base commune » qui relie les pratiques culturelles et religieuses afro-américaines à leur origine africaine. Or la perspective de Gilroy est fondamentalement différente, puisqu'elle ne prétend pas souligner les continuités entre la "diaspora » et la "Terre Mère ", mais la circulation de personnes, d'idées et de choses au sein de l'«Atlantique noir », dans une rencontre entre des réalités qui ne sont pas nécessairement les mêmes.

Le nouvel ouvrage de Lorand Matory constitue une remise en question et, en même temps, un prolongement des analyses de Paul Gilroy. Reprenant la principale critique formulée à l'égard du travail de Gilroy, Matory lui reproche d'avoir négligé les autres protagonistes de cette rencontre, en se concentrant dans son ouvrage sur les élites artistiques et intellectuelles afro-américaines aux ÉtatsUnis et leurs rapports avec l'Europe et l'Afrique. L'auteur veut replacer au centre de son analyse la «participation des Africains dans ce phénomène trans-territorial ", en remettant en cause l'idée défendue par Gilroy de la naissance d'une « histoire culturelle de la diaspora africaine » qui serait liée à la rencontre des Noirs nordaméricains avec les idées des Lumières (p. 289). Pour Paul Gilroy, l'esclavage, le racisme et la violence engendrée par le modèle esclavagiste représentent des moments-clés dans la constitution d'une " conscience moderne ». Il devient donc indispensable de réintégrer ce qu'il définit comme une "contre-culture de la modernité produite dans l'Atlantique noir " au sein des discussions sur la formation de la "modernité occidentale », deux facettes également importantes d'un même processus.

2. Philip D. Curtin, Seven Feierman, Leonard Thompson \& Jan Vansina, African History, Boston, Little \& Brown, 1978.

3. John Thornton, Africa and the Africans in the Making of the Atlantic World, 14001680, Cambridge, Cambridge University Press, 1992.

4. Robert Farris Thompson, Flash of the Spirit. African and Afro-American Art and Philosophy, New York, Random House, 1983. 
La critique de Matory fait écho à d'autres critiques développées dans des pays, comme le Brésil, qui se sont sentis exclus de cette histoire d'une "contreculture de la modernité ». Ainsi, Paulo da Luz Moreira ${ }^{5}$ (2004/2005), dans son compte rendu de l'édition brésilienne de l'ouvrage de Gilroy ${ }^{6}$, fustige l'absence de toute référence "à la relation asymétrique entre cultures noires du centre et de la périphérie du capitalisme mondial ", reprochant au texte de Gilroy de se cantonner « au monde anglo-saxon, européen et américain ». Ces critiques sont d'autant plus injustifiées que, dans la préface à l'édition brésilienne, P. Gilroy reconnait que la notion d'Atlantique noir gagnerait en profondeur en incorporant l'Atlantique Sud et ces formations culturelles, ce qui permettrait de sortir de la «camisole de force" du modèle nord-américain. Et s'il est vrai que l'auteur a concentré son analyse sur les artistes et intellectuels états-uniens, il n'a cessé de souligner l'importance d'une " histoire de la modernité reconstruite à partir du point de vue des esclaves $»$ (ibid. : 55) ${ }^{7}$.

Lorand Matory propose donc un changement de perspective qui met au centre de son analyse une classe d'entrepreneurs noirs brésiliens - marchands de produits africains - qui ont établi, au long des $\mathrm{XIX}^{\mathrm{e}}$ et $\mathrm{XX}^{\mathrm{e}}$ siècles, un réseau d'échanges fondé sur la circulation de marchandises, d'idéologies et de pratiques culturelles et religieuses. Son objectif principal est de resituer l'étude du candomblé dans une « diaspora afro-atlantique », en soulignant les processus transnationaux à l'origine d'une «culture noire atlantique». Pour cela, il utilise la métaphore du « dialogue » qui permet d'explorer les multiples liens entre Africains et « Africains Américains » (p. 291) ${ }^{8}$. L'objectif principal de l'ouvrage de Matory est ainsi de

5. Paulo da Luz Moreira, «Uma resenha de O Atlântico Negro, de Paul Gilroy », Revista literatura e cultura, 4(4), 2004-2005.

6. O Atlântico Negro. Modernidade e dupla consciência, São Paulo-Rio de Janeiro, Universidade Cândido Mendes-Centro de Estudos Afro-Asiaticos, 2001.

7. S'inspirant du travail de Gilroy, Stephan PALMIÉ, Wizards and Scientists. Explorations in Afro-Cuban Modernity \& Tradition, Durham-London, Duke University Press, 2002, a intégré l'histoire des esclaves et des Noirs cubains - et notamment de leurs avant-gardes intellectuelles dans l'analyse de ce qu'il appelle la "modernité atlantique ": "For what I am to demonstrate is that, far from designating even only typological opposites, the meanings associated with the terms Western modernity and Afro-Cuban tradition represent mere facets or perspectival refractions of a single encompassing historical formation of transcontinental scope. More for convenience's sake than for any (but the most obvious) analytic reasons, I call this formation Atlantic modernity. What I take this concept to refer to is, in part, a set of structural linkages that, since the early sixteenth century, transformed the Atlantic Ocean into an integrated geohistorical unit: an expanding theatre of human interaction defined by a vast and intricate web of political and economic relations objectively implicating actors and collectivities on three continents in each other's histories. More important, however, I also intend this term to designate a no less multiply determined, heterogeneous, and historically contingent aggregate of local discourses and practices reflecting on, engaging, and thereby both shaping and transforming this basic structural constellation " (ibid. : 15).

8. De façon étonnante, Matory utilise très souvent le terme "African-American ", très connoté idéologiquement dans la littérature nord-américaine, pour désigner les Noirs brésiliens. Cela lui permet de souligner leur "africanité » aux dépens d'une identité métisse, produit de l'histoire brésilienne, normalement mise en avant dans la littérature afro-brésilianiste. 
retracer toute une série de " dialogues transnationaux ", impliquant " des prêtres africains occidentaux, afro-brésiliens et afro-cubains, ainsi que des marchands d'esclaves européens et américains, des impérialistes européens, des nationalistes latino-américains et africains, des marchands noirs trans-atlantiques et une communauté internationale d'ethnographes »(p. 3).

Or, cette attention au dialogue entre différentes sphères sociales, présentée comme étant un des points principaux qui différencient l'approche de Matory de celle de Gilroy (p. 290), n'est pas sans rappeler la construction même du travail de ce dernier. On pourrait même dire que l'ouvrage de Matory constitue une réponse, à partir d'un agenda politique "African American », au travail de Gilroy. The Black Atlantic a été, en effet, une véritable bombe lancée dans les milieux afrocentristes nord-américains, puisque, pour la première fois, on introduisait une troisième dimension dans le rapport entre Africains et Africains Américains : l'influence européenne dans la pensée afro-américaine aux États-Unis. De plus, Gilroy menait là un projet hautement provocateur : penser l'expérience de l'esclavage et de « la rationalisation de la terreur dans la modernité » par rapport à l'Holocauste juif. Une audace impensable dans le milieu intellectuel très policé des États-Unis et inconcevable pour des militants afrocentristes particulièrement virulents contre les juifs nord-américains. Malgré cela, Gilroy juxtaposait dans son ouvrage la diaspora noire et la diaspora juive, afin de démontrer l'importance de la rupture et de la dispersion dans l'histoire de la modernité. De même, ses commentaires sur l'afrocentrisme n'étaient pas moins iconoclastes, le taxant d' " attitude provincialiste " ou, de façon plus adéquate, d' " américocentricité " dont les partisans "luttent fréquemment pour imposer leurs histoires au niveau du réseau de la diaspora et n'éprouvent aucune inhibition à revendiquer un statut spécial pour leur version singulière de la culture africaine » (Gilroy, 2003 : 252). Cela fait émerger, selon Gilroy, " une africanité fortement mythologisée, qui ellemême trouve son origine non pas en Afrique, mais dans une variété de l'idéologie panafricaniste produite tout récemment par l'Amérique noire » (ibid.: 123).

Lorand Matory, professeur au Département d'anthropologie et d' "AfroAmerican Studies » à la Harvard University, lui-même Afro-Américain marié à une Nigériane appartenant au sous-groupe yoruba des Ekiti, pouvait difficilement épouser une telle analyse ${ }^{9}$. Cependant, son ouvrage reprend plusieurs points développés dans le texte de Gilroy. Ainsi, l' « Atlantique noir » y est défini comme " a commercial, migratory, and discursive field » (p. 273), " a geographical focus,

9. Notons au passage qu'en dehors de l'affrontement idéologique entre un intellectuel African American états-unien, l'anthropologue africaniste Lorand Matory, et un autre intellectuel noir, le sociologue Paul Gilroy, né en Angleterre, on est ici confronté à la concurrence historique entre les deux grands centres universitaires américains : Harvard University et Yale University. Gilroy a travaillé jusqu'en juillet 2005 à Yale University, en tant que professeur de sociologie et directeur du Département d' "African American Studies ", pour rentrer en Angleterre, où il occupe depuis la chaire de théorie sociale Anthony Giddens à la London School of Economics and Political Sciences. 
an identity option, and context of meaning-making» (p. 274), une définition qui reprend, d'une certaine façon, les grandes lignes du travail de Gilroy. De même, l'importance accordée aux métaphores du "rhizome " et du " dialogue " sont inspirées, de façon claire (cf. p. 280), par le travail de Gilroy qui, par le biais du « chronotope du carrefour ", a souligné l'importance des échanges interculturels dans cet espace translocal. En définissant la tradition comme un méta discours qui permet le dialogue au sein de l'Atlantique noir, Gilroy écrit (2003: 262) : «Il ne serait pas absurde d'essayer de réserver l'idée de tradition à ces qualités minimales, évasives, dépourvues de nom, qui rendent possible ces conversations au sein de la diaspora ».

Les points de contact sont trop nombreux pour pouvoir en donner ici une liste exhaustive. Citons l'importance accordée à l'agency des acteurs noirs ; l'approche transnationale de l'univers afro-américain imposée par les échanges entre Afrique et Amériques, très forts depuis, au moins, le XIX siècle ; la circulation intense de personnes, marchandises et pratiques culturelles d'un côté à l'autre de l'Atlantique, même après l'abolition de l'esclavage. Matory remplace les marins et les voyageurs états-uniens qui, avec leurs allers et retours, ont donné vie, selon Gilroy, à l'" Atlantique noir ", par une classe marchande afro-brésilienne, très liée aux élites religieuses de certains centres de culte de Salvador de Bahia et engagée dans des échanges commerciaux avec le Nigeria et l'actuel Bénin. On retrouve là le même discours sur les élites noires, dont l'agencéité (agency) au regard des masses anonymes pourrait sembler parfois surévaluée. Ainsi, Matory (p. 42), en soulignant les ressemblances entre les Lucumís cubains, les Nagôs brésiliens, les Nagôs haïtiens, les Nagots de l'Afrique occidentale française et les Yoruba de l'Afrique occidentale britannique, voit dans cette "unité » le produit "des intérêts d'une puissante classe d'“entrepreneurs noirs" de l'ethnicité ". Ce seraient donc ces voyageurs entre les Amériques et l'Afrique qui auraient aidé à créer " la culture et la religion traditionnelle yoruba ", en les imposant en tant qu'« exemple normatif de la culture africaine dans le Nouveau Monde » (p. 50).

L'originalité du travail de Matory réside dans ce changement de focale, qui met au premier plan les acteurs afro-brésiliens de cette rencontre entre Afrique et Amériques. Il critique une certaine tendance, au cœur des études afro-brésiliennes, à souligner le poids de l'influence des élites intellectuelles brésiliennes - qu'il désigne par les termes "Euro-Brazilian elites » en dépit du métissage évident d'un grand nombre d'intellectuels, surtout les Bahianais - dans " l'invention des traditions " afro-brésiliennes : "In this model, African-American cultural debts to Africa and agency in the construction of their own traditions are either marginalized or denied, and the power of Euro-Brazilian sponsors to reshape Afro-Brazilian religious practice in their own oppressive interest is emphasized" (p. 14). Cette critique a déjà été formulée par plusieurs auteurs. Personnellement, je n'ai pas cessé de souligner la nécessité de réintégrer l'agency de certains leaders religieux du candomblé qui ont souvent joué un rôle très important dans 
l'établissement d'un modèle de tradition pour l'ensemble des maisons de culte de candomblé et dont le discours a souvent été reproduit de façon acritique dans les écrits anthropologiques (voir Capone, La quête de l'Afrique dans le candomblé. Pouvoir et tradition au Brésil, Paris, Karthala, 1999). S'il faut parler d'un dialogue, alors ce dialogue ne peut pas être exclusivement entre "AfroBrésiliens » et "Africains ", comme le suggère notre auteur, mais aussi, et surtout, entre élites religieuses et élites intellectuelles brésiliennes. Et cela indépendamment de la couleur de peau de leurs membres. Dans l'analyse du candomblé proposée par Matory, cette religion se métamorphose en patrimoine ethnique des descendants d'esclaves, alors que, depuis très longtemps, le candomblé n'est plus une « religion ethnique ", mais une religion "universelle ", ouverte à tous, indépendamment de l'origine, du sexe, de la couleur de peau des uns et des autres.

Malgré cette lecture de la société brésilienne selon des catégories nordaméricaines, l'ouvrage de Matory constitue une importante remise en question de certaines approches classiques dans le domaine afro-américaniste. Pour cet auteur, ce qui, pendant très longtemps, a été considéré comme «survivances passives " de la culture africaine dans les Amériques, n'est autre que le produit d'une " ongoing human agency in a circum-Atlantic field» (p. 35). Cette agency ne peut se déployer qu'à travers ce que l'auteur appelle un "Afro-Atlantic dialogue ». L'ouvrage explore, spatialement et temporellement, ce dialogue entre Africains et "Afro-Brazilians", allant au fil des chapitres de la moitié du $\mathrm{XIX}^{\mathrm{e}}$ siècle jusqu'au début du XXI ${ }^{\mathrm{e}}$ siècle. L'objectif principal de Matory est de montrer comment l'idée de "pureté », au cœur de la pratique du candomblé, constitue un des produits de cette rencontre et de ces dialogues transcontinentaux.

L'hypothèse la plus intéressante est, sans aucun doute, l'idée d'une influence directe des « élites de la diaspora » sur leur terre mère. Reprenant les critiques de l'interprétation du transnationalisme en tant que phénomène nouveau, Matory affirme, de façon hautement provocatrice, que « les diasporas créent leurs mères patries" (p.3), produisant toute une série de nouvelles identités ethniques en Afrique occidentale. C'est le cas de l'identité ethnique "Djedji » en Afrique de l'Ouest et de son correspondant religieux brésilien, la «nation jeje » de candomblé, tous deux produits par les allers et retours d'Afro-Brésiliens à travers l'Atlantique, analysés dans le chapitre 2 . ̀̀ travers cet exemple, il se propose de repenser le rapport entre "nations " et "transnationalisme ", montrant comment la dimension transnationale ne préfigure pas la mort de la nation. Comme il l'écrit dans son introduction: "lifeways, traditions, and the social boundaries they substantiate endure not despite their involvement in translocal dialogues but because of it " (p. 4). L'influence des "Brésiliens ", les esclaves affranchis brésiliens rentrés en Afrique et devenus rapidement une communauté de "marchands et administrateurs au service des colonisateurs européens " (p. 84), est ainsi à l'origine du développement d'une «nation de culte» du 
candomblé, appelée Jeje Marrim (ou Màxí), à Bahia ${ }^{10}$. Ce sous-groupe, faisant partie de la «nation jeje ", est aujourd'hui devenu le modèle dominant de cette "nation» de culte, dont le déclin à la fin du XIXe siècle a été suivi par un essor des maisons de culte jeje dans les années 1930. Cette renaissance serait le résultat des voyages, à travers l'Atlantique, de cette classe de marchands afro-brésiliens qui "share with many other trade diasporas and social elites a concern with racial and cultural "purity' as an index of membership and rank" (p. 91).

Le pouvoir de transformation attribué à cette classe marchande est très important. Si l'influence de marchands de "biens ethniques " associés à la pratique du candomblé, comme la noix de cola, les pagnes tissés (panos da costa) et le beurre de karité, est déjà avérée pour le cas de Martiniano do Bonfim et Felisberto Sowzer, le rôle joué par d'autres marchands dans l'établissement de "nations de culte » similaires au Brésil et à Cuba semble être beaucoup moins évident ${ }^{11}$. Cette classe marchande partagerait avec les returnees du Nigeria un même souci de "pureté " qui les différencierait de la masse des Africains. Et c'est en cela que réside le principal paradoxe de cette histoire transnationale : les anciens esclaves retournés en Afrique ne se pensent pas en tant que membres arrachés aux peuples africains, mais en tant qu'élites « illuminées » et " évoluées » grâce à leur proximité avec la culture et le pouvoir des Blancs. Les "Brésiliens » au Bénin ont ainsi sauvegardé tous les signes distinctifs de leur origine transatlantique : ils ont préservé leurs croyances catholiques, leurs festivités brésiliennes et la culture culinaire afro-brésilienne comme autant de preuves de leurs brésilianité. Comme les returnees nigérians, ils se considèrent supérieurs à leurs compatriotes indigènes, se comportant comme les «élites créoles dans le Nouveau Monde »: "Their position in the colonial hierarchy placed them disproportionately in a position to speak for their African compatriots and to assume leadership in the independent nation-states of Dahomey/Bénin and Togo (not to mention the parallel Nigerian case) 》 (p. 85).

Ce rapport de domination vis-à-vis des indigènes a aussi entraîné des formes de sentir et de penser la pratique religieuse qui sont très éloignées de la réalité africaine. Ainsi, Matory montre, à partir de ses recherches dans la ville de İgbòho, comment la pureté n'est pas un critère important dans la pratique religieuse des Yoruba au Nigeria. Pour lui, le succès «disproportionné et anormal » d'un

10. Dans ce chapitre, l'auteur semble parfois confondre la notion d'État-nation avec celle de "nation " dans le candomblé. S'il y a une base commune entre ces deux notions, c'est bien l'idée d'une "communauté imaginée " (Benedict Anderson, Imagined Communities, LondonNew York, Verso, 1983). Cependant, les formes d'appartenance à ces communautés ne sont pas les mêmes, puisque un initié dans la «nation » jeje ou ketu ne se pense pas - ni n'est pensé en tant que membre d'un groupe ethnique correspondant africain. Dans le candomblé, l'identité religieuse n'équivaut en aucun cas à une identité ethnique.

11. C'est le cas de Joaquim d'Almeida, un returnee brésilien qui possédait des esclaves à La Havane et dans l'État de Pernambouc au Brésil, que Matory considère à l'origine des liens entre "the emergent Màxi politico-cultural traditions of Cuba, Brazil, and the West African Coast» (p. 94). 
idiome rituel «puriste» dans le candomblé brésilien serait dû à ses "affinités électives " avec deux facteurs: "the condition of diaspora and the role of merchant interests in making diasporas endure as communities " (p. 116). Les marchands avaient besoin de présenter leurs marchandises comme "purement" africaines pour pouvoir les imposer sur un marché où tout ce qui est africain acquiert automatiquement de la valeur, monétaire et symbolique. La "pureté africaine " devient ainsi, pour Matory, "a shared symbolic meta-logic available for use in cooperative or competitive projects" (p. 144).

Mais, s'il souligne à juste titre " the impure roots of purity» (p. 121), Matory ne laisse pas pour autant de reproduire un certain discours sur le candomblé qui fait de cette "pureté " et de ses racines, dans un passé immémorial, les critères indispensables d'une hiérarchie de pouvoir au sein des différentes "nations de culte ». Très souvent, l'auteur épouse le discours - the narrative - de l'Axé Opô Afonjá, maison de culte censée être parmi les plus «traditionnelles » de Salvador de Bahia. Ainsi, ce terreiro est défini comme étant "the most purist and Africacentered of Bahia's "great houses" " (p. 172) et les maisons de la «nation ketu (ou quêto)/nagô » sont présentées comme "plus africaines » et "plus dignes de respect " que les maisons de "nation congo-angola" (p. 24) ${ }^{12}$. Ainsi, malgré son souci de montrer comment la "mémoire culturelle ", les "survivances" et la «tradition", en Afrique ou dans la diaspora, sont toujours le résultat de rapports de pouvoir, soumis à une négociation et à une re-création stratégique (p. 70), Matory se concentre sur les gagnants de cette lutte pour l'hégémonie dans le champ religieux afro-brésilien, reléguant les vaincus (les terreiros angola) à leur statut de dominés.

D'une certaine façon, Matory reprend ici la critique de Gilroy contre le discours d'inspiration nationaliste qui voit dans l'Afrique l'origine d'une culture noire " pure ", pour montrer que si l'idée de " pureté » a été formulée en Afrique par un mouvement nationaliste comme celui de la Lagosian Cultural Renaissance, à l'origine du nationalisme nigérian, elle arrive au Brésil grâce à la médiation d'une classe marchande "translocale ». L'idée de pureté est ainsi stratégiquement déployée pour servir les intérêts, politiques et commerciaux, d'élites diasporiques «redevenues» africaines. Mais ce qui est gommé dans l'analyse de Matory, c'est justement l'importance de l'influence européenne sur ces élites de returnees. John Peel ${ }^{13}$ a bien montré le rôle des agents missionnaires dans la naissance du nationalisme yoruba. Il ne faut pas oublier en fait que les returnees

12. En parlant des images filmées dans les maisons de culte de candomblé, il écrit : « Hence, it is precisely the houses that are least preoccupied with "African purity" that are most likely to call themselves "Angola" and to allow filming" (p. 25). Sur ce lien entre "pureté " et candomblé nagô, opposé à la "dégénérescence " des pratiques des "nations bantu ", cf. Stefania CAPONE, 1999, op. cit. et 2000, "Entre Yoruba et Bantou : l'influence des stéréotypes raciaux dans les études afro-américaines ", Cahiers d'études africaines, 157, p. 55-77.

13. John D.Y. Peel, Religious Encounter and the Making of Yoruba, Bloomington-Indianapolis, Indiana University Press, 2000. 
Krios ou Saros - anciens esclaves libérés par les navires anglais et débarqués en Sierra Léone - ont été éduqués dans des écoles missionnaires protestantes et sont devenus les premiers agents missionnaires indigènes une fois retournés au pays. L'ouvrage de Peel souligne l'importance du dialogue entre les différents agents engagés dans cette "rencontre religieuse " (religious encounter) où l'influence européenne est décisive ${ }^{14}$. Le rôle des agents missionnaires africains dans la construction d'un nationalisme culturel est avéré et ces africains "évolués " ont activement participé à la formation d'une élite lettrée yoruba. C'est donc à ces élites chrétiennes, de formation européenne, que les returnees et les marchands afro-brésiliens auront à faire. L'effacement de ce troisième terme dans la rencontre entre Africains et membres de la diaspora est peut-être le point le plus faible de l'argumentation de l'auteur.

Cette prise de position, plus idéologique que scientifique, est aussi à l'origine de plusieurs contradictions. Ainsi, si Matory critique certains auteurs brésiliens qui défendraient la thèse d'une soumission passive des élites du candomblé aux élites "Euro-Brazilian ", il réintroduit cette même idée lorsqu'il discute, dans le chapitre 5, l'établissement d'un modèle de tradition lié à un supposé matriarcat dans les maisons de culte de Salvador. Selon Matory, le "monopole féminin » dans le candomblé serait le résultat d' « efforts transnationaux et de raisons nationalistes » (p. 187). D'un côté, il y aurait une "perspective féministe occidentale ", véhiculée par le travail de l'anthropologue nord-américaine Ruth Landes ${ }^{15}$ et "son imagination d'une communauté transnationale de femmes» (p. 195); de l'autre, les efforts d'une "bourgeoisie euro-brésilienne » pour acquérir " une position d'estime au sein de la culture transnationale du nationalisme [sic] " (p. 187). Cette dernière affirmation n'est pas argumentée et ne semble pas se justifier lorsqu'on connaît la polémique engendrée par l'ouvrage de Ruth Landes au Brésil, notamment les critiques féroces formulées par le plus important des spécialistes des religions afro-brésiliennes de l'époque, Arthur Ramos. Par ailleurs, comment un livre sur le matriarcat dans les maisons de culte "traditionnelles " de candomblé, comme celui de Landes, qui n’a été publié pour la première fois au Brésil qu'en 1967, aurait eu le pouvoir d'imposer ce type de paradigme à l'ensemble de la "prêtrise du candomblé depuis 1930 » (p. 187) ? Dans cette partie de son ouvrage, Matory inverse ainsi complètement son hypothèse de départ, en mettant en avant un supposé "pouvoir de transformation » des élites "eurocentric » et en sous-estimant l'agency des mères de saint des maisons ketu de Salvador, dont Ruth Landes avait reproduit le discours hégémonique. En réalité, dans les études afro-brésiliennes, l'impact de cette théorie d'une prédominance féminine dans le leadership du candomblé ne devient effectif qu'à partir des années quatre-vingt, avec les travaux de Peter Fry et Patricia Birman.

14. Voir à ce sujet la note critique d'André MARY ("L'invention chrétienne de l'identité yoruba. Les racines missionnaires d'une nation africaine ", Archives de sciences sociales des religions, 124, 2003, p. 49-61) à propos de l'ouvrage de J.D.Y. Peel.

15. Ruth Landes, The City of Women, New York, MacMillan, 1947. 
Malheureusement, Lorand Matory n'analyse pas la littérature récente sur la question du genre dans le candomblé, ne citant aucune publication éditée sur ce thème depuis 1995, date de son dernier séjour sur le terrain au Brésil ${ }^{16}$.

Malgré ces quelques faiblesses de l'argumentation et plusieurs imprécisions dans ses données ${ }^{17}$, l'ouvrage de Matory a le mérite d'offrir une nouvelle lecture - à travers le prisme afro-américain - de ces phénomènes religieux. Son livre est donc, sans aucun doute, une importante contribution à l'écriture d'une histoire des élites afro-bahianaises. De plus, il ne s’intéresse pas à la continuité des pratiques culturelles - les Africanismes - mais aux processus historiques qui ont créé ces continuités, estompant officiellement les innombrables discontinuités. Cela lui permet de souligner l'affirmation stratégique de l'africanité dans une arène transatlantique qui est, à la fois, commerciale, culturelle et politique.

Son travail trouve ses limites dans une conceptualisation de la diaspora comme "communauté » soudée, au sein de laquelle il n'y aurait pas de contrediscours sur la légitimité et la pureté des pratiques religieuses. Cette approche "politiquement correcte" met en avant l'unité et non pas la fragmentation de la «Black Atlantic». Le regard nord-américain sur les matériaux brésiliens est ce qui pose le plus de problèmes, surtout dans une ville comme Salvador, ville " africaine " par excellence, où subsistent des divisions internes avec l'existence de classes socialement "blanches ». Or, pour le regard afro-américain, les Bahianais apparaissent tous comme des "noirs» et le candomblé devient une religion " africaine ", puisque pratiquée par des "afro-descendants ». Cela ne correspond pas nécessairement à la société brésilienne, où les catégories " raciales " sont aussi fortement dépendantes de critères sociaux. Ainsi, si Lorand Matory reconnaît en passant que «les Européens, les Euro-Américains et les Amérindiens sont profondément concernés par le dialogue afro-atlantique " (p. 271), cette pluralité de voix disparaît lorsqu'il s'agit de penser les rapports entre «Afro-Brésiliens » et "Africains ». Ce qui devient de nos jours toujours plus difficile est de délimiter ce qui est exclusivement " noir » dans cette "Black Atlantic ". S'il y a eu un dialogue à l'origine de cette configuration transnationale, il n'a pu se faire sans mobiliser tous les acteurs en présence. Sans la pluralité de voix qui est à la base du modèle proposé par Paul Gilroy, la prégnance même du concept d' "Atlantique noir " pourrait perdre de sa valeur et ne produire que des lectures partielles de cette rencontre transcontinentale.

16. Matory a en effet passé, en tout, quatorze mois sur le terrain au Brésil, en 1987, 1992 et 1995-1996 (p. 33).

17. Par exemple, la date de l'indépendance du Brésil qui, de 1822, devient 1922 (p. 86) ou le médecin légiste Raymundo Nina Rodrigues, précurseur des études afro-brésiliennes, qui est présenté en tant que psychiatre (p. 167). 